\title{
Two cases of penile strangulation: varied presentations and vastly different outcomes
}

\author{
Mayank Agrawal, Venkat Arjun Gite* ${ }^{*}$ and Prakash Sankapal
}

\begin{abstract}
Background: Penile strangulation by various metallic and non-metallic objects is a true urological emergency that requires prompt emergency management. The cases in this report will help in highlighting the varied presentations one can face in the emergency department. Management of such cases at times needs out-of-the-box thinking and improvised skills as resources to remove the foreign body are often scarce within the hospital.

Case presentation: We present two such cases of penile strangulation in adult patients. Both patients presented to us in the emergency department, one with a large metallic cone and another with a rubber band constricting their penises. Both the patients had hugely different grades of injuries and were managed accordingly. Both the patients required different methods to remove the constriction objects as per the need of the situation. One of the patients required total penectomy with permanent perineal urethrostomy; however, in the other case, we were able to save the penis.

Conclusion: Penile strangulation needs urgent medical attention and timely removal of the offending object. Grade of injuries and complications are directly proportional to the type of object and the duration of the strangulation. The non-metallic objects are easy to cut and remove. However, one should be aware of the challenges and the complications in managing metallic foreign bodies which at times may need out-of-the-box thinking, like use of motorized cutting tools.
\end{abstract}

Keywords: Penile strangulation, Penile gangrene, Metal cone, Rubber band, Case report

\section{Background}

Penile strangulation is a urological emergency in true sense which can have drastic and life-changing consequences. The causes may be varied: devices applied on the penis for self-stimulation and sexual gratification, pranks that went horribly wrong, or devices applied for external urinary drainage to manage urinary incontinence or enuresis. These patients often have associated psychiatric disorders $[1,2]$.

The objects reported are metal rings, bands, pipes, bottles, threads of metal or plastic or latex of all shapes and sizes $[3,4]$. These objects have constriction force on the penis which hampers blood and lymphatic flow, resulting

*Correspondence: balajigite@yahoo.com

Department of Urology, Grant Medical College and Sir JJ Hospital, Mumbai 400008, India in strangulation. Early removal of the constriction object with the restoration of blood supply is paramount as delay in treatment may result in irreversible damage to the penis in the form of gangrene $[2,5]$.

We report two such cases highlighting the different clinical presentations and challenges in the management of penile strangulation.

\section{Case presentation}

\subsection{Case 1}

A 40-year-old man presented to the emergency department with a history of metal cone insertion over his penis 7 days ago. The patient slipped it over his penis for self-sexual stimulation beyond the glans. Despite his best efforts, he could not take out the metal cone. He could not reveal his problem to his family due to fear of embarrassment. After all his attempts to remove 
the metal cone failed, he finally decided to seek medical help in view of increasing penile swelling. On examination, the patient was hemodynamically stable, conscious, oriented, not in any pain but anxious and fearful. On local examination, a metal cone encircling the proximal penile shaft was found with dimensions of $4.5 \mathrm{~cm} \times 3 \mathrm{~cm} \times 0.3 \mathrm{~cm}$. There was polythene underneath the metal cone which patient had placed to facilitate the easy sliding of the penis (Fig. 1). There were gross oedema and congestion of the penile shaft and prepuce distal to the metal cone. There was no blackish discolouration or signs of necrosis of distal penile shaft. The glans penis and external urethral meatus were not visible due to preputial oedema. There were foul-smelling pus and slough underneath the metal cone as a result of necrosis of the skin due to the strangulating effect of the metal cone. There was no evidence of any urethro-cutaneous fistula. The patient had a poor urinary stream but was able to empty his bladder. On per abdominal examination, bladder was nonpalpable. After visual and physical examination of the patient's condition, it became clear that the patient will need surgery to remove the metal cone and examination of the underneath penis and soft tissue viability. Basic blood investigations were sent, and anaesthesia fitness was obtained for emergency surgery. A psychiatric evaluation was done which revealed that the patient was suffering from depression.

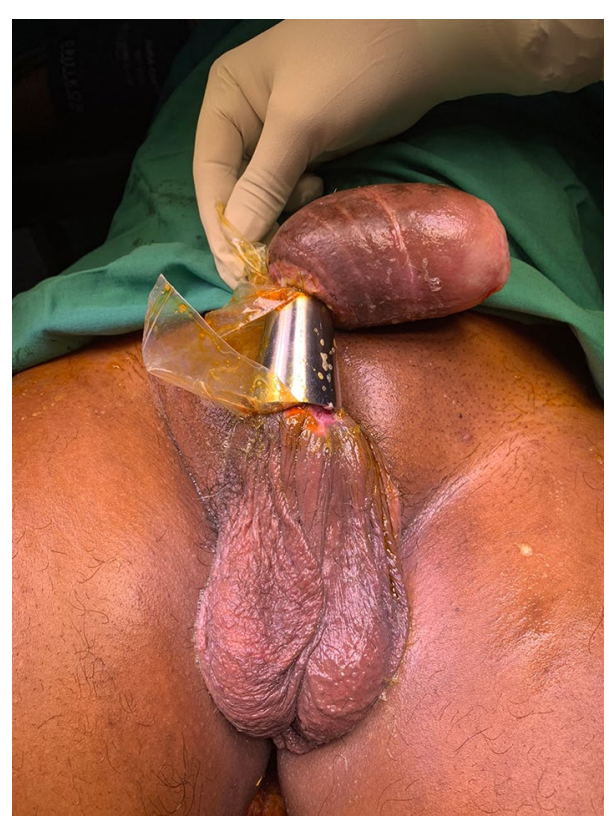

Fig. 1 Metal cone encircling the proximal penile shaft with polythene underneath it. Gross oedema of the penile shaft and prepuce distal to the metal cone is evident

\subsubsection{Procedure}

Consent was taken for surgery including partial/total penectomy with perineal urethrostomy, depending upon the condition of underneath tissue. An electric metal cutting drill was arranged from outside the hospital. The patient was taken under general anaesthesia. Before cutting the cone using an electric drill (BOSCH GWS 600 professional angle grinder), a metal ruler and a scalpel handle were introduced underneath the metal cone to prevent iatrogenic injury to the penis and surrounding soft tissues (Fig. 2a, b). Protective gearings were born by the operating team. Metal cone was cut through longitudinally at 12 o'clock and at 6 o'clock (180-degree opposite to each other). Continuous cold-water irrigation was done to avoid the burn injury to the underneath skin by an increase in temperature of the metal cone. Cone was removed in two pieces (Fig. 2c). There was a constriction ring formed at the base of the penis. Release incision was given dorsally over the prepuce and the penile skin (Fig. 3a). There was active bleeding along with ooze of collected interstitial fluid indicating the viability of the distal penile tissue. The collected interstitial fluid was squeezed out. The viability of underneath soft tissue was assessed and confirmed. The glans was normal pink in colour. External meatus was normal. There was no bleeding per urethra. The constriction ring made by dead and necrotic skin at the proximal penile region was excised circumferentially (Fig. 3b). The incised skin was sutured on itself using a 3-0 polyglycolic suture. 14 Fr silicone Foley catheter was placed (Fig. 4a, b). Haemostasis was confirmed, and the dressing was done.

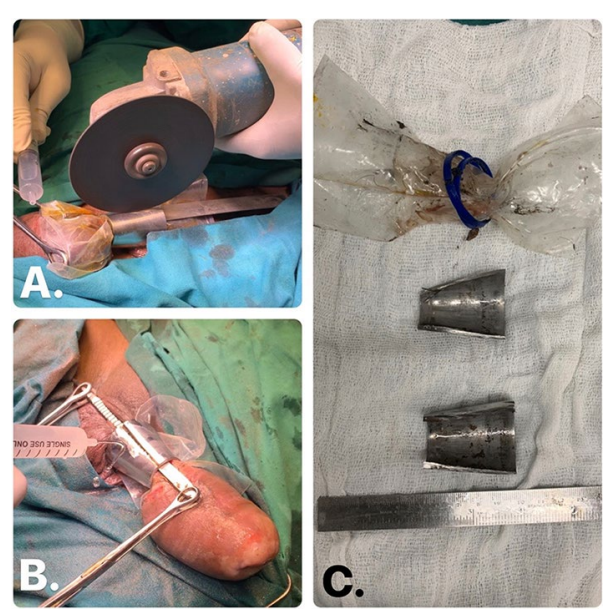

Fig. 2 Metal cone was cut using an electric drill, and a metal ruler was introduced underneath the metal cone to prevent the iatrogenic injury. a Scalpel handle and continuous cold-water irrigation used to avoid the iatrogenic injury. b Metal cone was cut at two places, 180-degree opposite to each other, and was removed in two pieces 

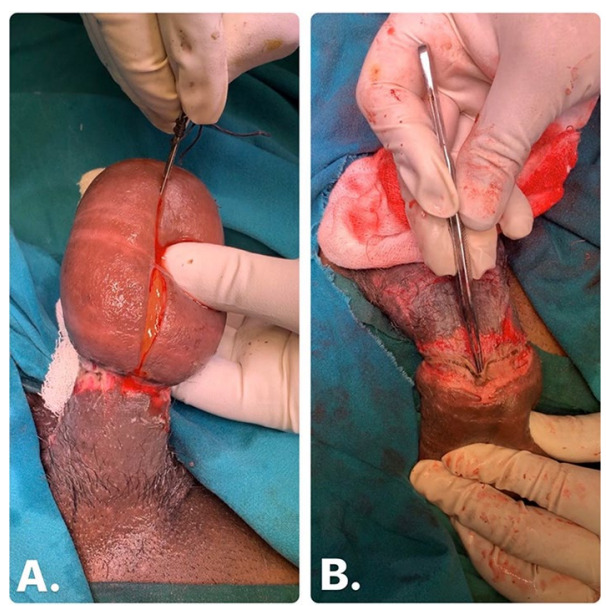

Fig. 3 a Release incision was given on the dorsal aspect of the penis, active bleeding along with ooze of collected interstitial fluid indicating the viability of the distal penile tissue. $\mathbf{b}$ Constriction ring formed at the site of metal cone made up of dead and necrotic skin was excised circumferentially
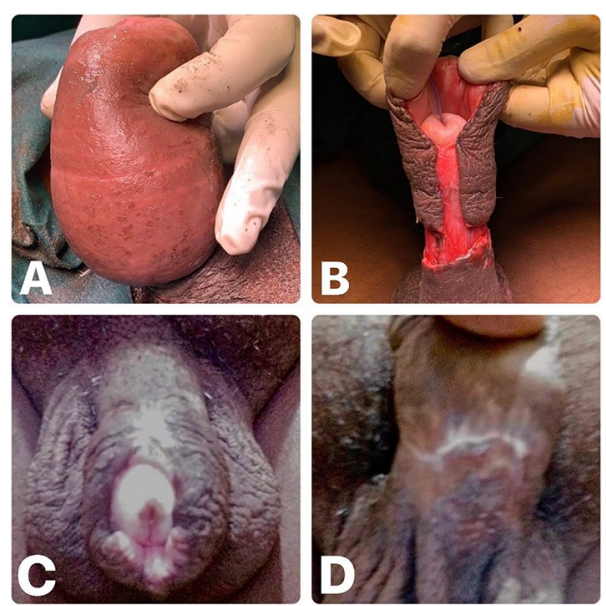

Fig. 4 a After removal of the metal cone. Penis was grossly oedematous with constriction near the base of the penis. b Penile oedema was reduced considerably after dorsal release incision was made along with the excision of the constriction ring. Glans penis and external meatus were normal. c Dorsal aspect of the penis with healed surgical site over the shaft, normal glans and normal external meatus at 3-month follow-up. $\mathbf{d}$ Ventral aspect of the penis with healed suture line at 3-month follow-up

In the post-operative period, patient was managed with antibiotics and daily dressing. A psychiatric reevaluation was done, and the patient was started on antidepressants and counselling was done. Penile oedema subsided gradually. After 7 days, suturing of the penile wound was done to close the release incision site. The catheter was removed, and the patient was discharged on post-operative day 14 . The patient was followed up at 4 weeks, and the wound had healed well. At 3-month follow-up, the patient had no urinary symptoms or erectile dysfunction or priapism (Fig. 4c, d). The patient is regular with his psychiatric follow-up and has resumed earning his livelihood.

\subsection{Case 2}

A 65-year-old man, chronic alcoholic, known diabetic presented to emergency with complaints of penile swelling and urinary incontinence for the last 1 week. There was no history of trauma. On examination, the patient was hemodynamically stable. Local examination revealed ulceration over the penile shaft and glans with oedematous and dusky discolouration of the penis (Fig. 5a, b). Penis was covered in slough. There was no active bleeding from the ulcerated part. Glans and distal penile shaft showed features of gangrene in the form of blackish discolouration. A constriction rubber band buried in slough at the base of the penis was found. There was considerable loss of both corpora cavernosa and spongiosum at the site of constriction, and an only thin rim of corporeal tissue remained underneath the rubber band. Ventrally, at the site of strangulation, there was a urethra-cutaneous fistulous with urine leaking from it. The patient told that he had urinary problems for which he was using a condom catheter. He often applied a rubber band to avoid slippage of the condom catheter, but for the last 2 weeks, he had started using a diaper instead of a condom catheter. He probably had forgotten to remove the rubber band from his penile shaft post-removal of the condom
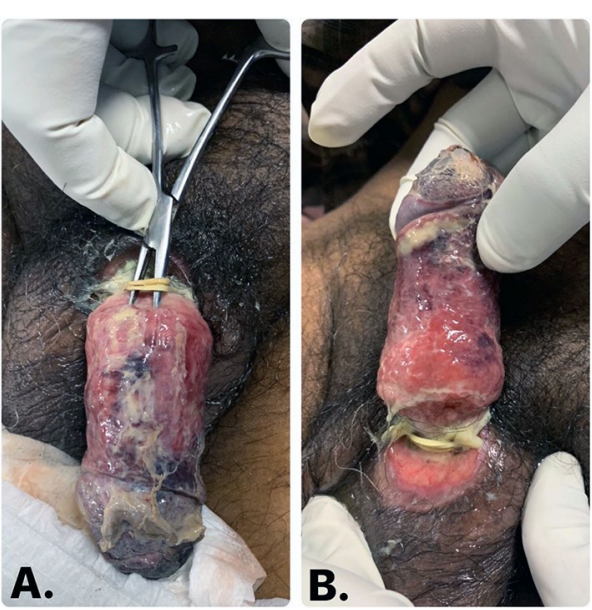

Fig. 5 a Glans and distal penile shaft were gangrenous, covered in slough with a constricting rubber band found at the base of the penis, buried in slough. $\mathbf{b}$ There was considerable loss of both corpora cavernosa and spongiosa at the site of constriction with urinary leak 
catheter. Penile Doppler did not show colour flow distal to the site of constriction.

\subsubsection{Procedure}

Emergency anaesthesia fitness was obtained, and consent for total penectomy with permanent perineal urethrostomy was obtained. The strangulating rubber band was cut with the help of scissors. Suprapubic catheterization (SPC) was done for urinary diversion along with total penectomy and permanent perineal urethrostomy. In the post-operative period, the patient was managed with daily dressing, anti-inflammatory, and antibiotics. Blood sugar was controlled. Per urethral catheter (PUC) was removed on post-operative day 14, and SPC was clamped. The patient voided well. SPC was removed after another 2 days. At 6-month follow-up, the patient had no urinary symptoms.

\section{Discussion}

Penile strangulation is a rare clinical condition that was first reported in 1755 [6]. They occur as a result of a constriction force of the object applied over the penile shaft. In adults, the common reasons are sexual stimulation, prolonging erection, pranks, treatment of incontinence and phimosis $[3,7]$. However, in kids, the main reason to place such objects is for managing enuresis $[2,8]$. Likewise, there are a plethora of objects which have been reported in the literature as a cause of penile strangulation like metallic rings, tubes, rings, plastic bottles, rubber bands, rubber strings, hair, threads $[3,5]$.

These objects when applied over a flaccid penis causes obstruction of venous and lymphatic outflow, resulting in the penile engorgement. Due to the oedema and increase in the girth of the penile shaft, the applied object which was placed easily before is now difficult to remove. As the pressure increases because of the lymphatic obstruction and venous congestion, the continuous constriction force results in compartment syndrome type situation. This eventually leads to obstruction of the arterial inflow to the distal part of the penis, resulting in strangulation [15]. If the offending object is not removed timely, then the strangulation would lead to soft tissue ischaemia, necrosis, local infection, and gangrene $[1,3,4]$.

In 1991, Bhat et al. graded these kinds of injuries according to the increasing severity [2].

Grade 1 Oedema of distal penis. No evidence of skin ulceration or urethral injury.

Grade 2 Injury to skin and constriction of corpus spongiosum but no evidence of urethral injury. Distal penile oedema with decreased penile sensation.

Grade 3 Injury to skin and urethra but no urethral fistula. Loss of distal penile sensation.
Grade 4 Complete division of corpus spongiosum leading to urethral fistula and constriction of corpus cavernosum with loss of distal penile sensation.

Grade 5 Gangrene, necrosis, or complete amputation of distal penis.

Most of the patients delay in seeking medical attention due to fear of social embarrassment or at times neglect the problem and seek medical help after failed self-attempts, as seen in our cases [5, 7]. The common complaints at presentation are penile swelling, pain at the local site, or difficulty in passing urine. The delay in presentation results in penile swelling out of proportion to the inner diameter of the object stuck, thus making the task of removing the foreign body even more difficult. The prolonged placement and delayed removal of these objects are likely to cause high-grade injuries $[1,4,5]$.

The evaluation of such patients should include thorough history taking including the duration for which the object is stuck. History about any substance abuse or any previous/ongoing psychiatric illness should be obtained. [1, 2, 4, 9-11]. The patient should be specifically asked about the voiding difficulty as urethra might be compressed because of the penile strangulation $[2,5]$.

Local assessment should be done by recording skin temperature, skin colour, penile sensation, and pulsation distal to the strangulation to assess arterial insufficiency [2]. The penis should be examined for any urinary leak from the ventral aspect as it can happen in case of the development of urethra-cutaneous fistula due to pressure effect. Skin ulceration and local infection can be present as a result of skin ischaemia [4]. Ultrasound Doppler can be used to assess the vascularity in the case of equivocal clinical findings $[2,5]$. Urinalysis and urine culture are also recommended $[4,5]$.

The early removal of the constriction device and restoration of venous and lymphatic drainage and arterial inflow is paramount $[1,8]$. It helps in preventing ischaemic injury to the penis and urethra. Removal of the offending object can be a real challenge due to surrounding tissue oedema. Bhat et al. in their study divided the injuries based on the type of offending objects, into the metallic and non-metallic groups. They concluded that the non-metallic objects are easy to remove, but the injury caused by them is severe, as they are thin and sharp [2]. In 2008, Silberstein et al. also reported that higher-grade penile injuries are more frequently sustained by non-metallic objects due to the more elastic properties of these items and their greater propensity to exert pressure on the penis and thus create more injury [5]. As per grading system proposed by Bhat et al., in our study, the first case had grade 2 injury by a metallic cone which required the use of an electric drill for its removal and the second case had grade 4 injury by a rubber band 
which was cut with the help of scissors and ultimately required total penectomy with permanent perineal urethrostomy. These findings were in correlation with the findings of Bhat et al. and Silberstein et al. [2, 5].

Apart from the material of the object, other factors to be considered before taking the patient for surgery are size, length, and thickness of the object, the grade of surrounding tissue oedema, the grade of injury, and availability of the equipment $[1,2,5]$.

There are a variety of techniques described in the literature like aspiration, string method, cutting devices, and degloving surgeries $[1,2,4,7,8,12]$. The cutting technique is by far the most used intervention [5]. The various tools used for the same are either non-electric or electric, viz. orthopaedic equipment, ring cutters, metal saws, hammer, chisel, drills, etc. The non-electric cutting devices are easy to use but require strength and are best reserved for non-metallic items, small metal rings or wires at the most $[4,5,7]$. On the other hand, electric cutting devices are high-energy-driven tools. They are of greater help in removing large and thick metallic objects, but their handling is difficult and requires utmost care and safety precautions to avoid iatrogenic injury to the patient and the operating team members $[1,4,5,7,13]$.

Use of protective gear for the team members is advisable $[1,4]$. Placement of a metallic object underneath the foreign body before using an electric drill helps in minimizing the risk of iatrogenic injury $[4,5,13]$. We used a scalpel handle and metallic scale in our case for this purpose. On similar lines, we used continuous cold irrigation while cutting to prevent heat injury to the underneath tissue $[5,13]$. When it comes to sawing the rings or cones, they should be cut at two places, 180 degrees opposite to each other for easy removal $[4,5]$.

Post-removal of the offending object, the underneath soft tissue and skin colour change must be examined for tissue viability. Post-removal of the constriction device as the blood supply is restored, skin colour returns to normal. This points towards the viability of the underneath soft tissues. In case of doubt, intra-operative penile colour Doppler can be helpful.

Associated injuries to the skin, corporeal bodies, spongiosum, and urethra must be evaluated. Any necrosed soft tissue or constriction band formed must be excised. Skin grafting might be necessary if the primary wound is too large to be closed primarily. In the case of urethral injuries or irreversible penile ischaemic injuries, urinary diversion in the form of suprapubic catheterization or perineal urethrostomy might be needed $[2,4,5]$.

The goal of the management is the early and safe removal of the foreign body and management of associated injuries. However, there are no standard guidelines to manage such cases due to varied presentations and in most cases the surgeon must resort to improvisation which may entail arranging machinery from outside the hospital [1]. In one of our cases, we had to arrange for the electric metal cutting drill from a nearby plumber.

In post-operative period, the patient should be closely monitored and examined for resolving oedema or any signs or symptoms of post-operative ischaemic injury. It should be ensured that the patient does not have any difficulty in voiding urine. Along with it, pain management, psychological evaluation, and counselling are necessary $[1,2,5]$.

Post-operative complications are more common in higher-grade injuries as reported by Bhat et al. [2]. Follow up is advised to evaluate and manage long term complications which may arise like urethra-cutaneous fistulas, urethral stricture, lymphoedema, skin necrosis, erectile dysfunction, and priapism $[2,4,5,7]$.

\section{Conclusion}

Penile strangulation is a rare but true urological emergency which needs urgent medical attention and timely removal of the offending object which can help in avoiding irreversible neuro-vascular damage to the penis and urethra.

Grade of injuries and complications are directly proportional to the type of object and the duration of the strangulation. The non-metallic objects are easy to cut and remove.

However, one should be aware of the challenges and the complications in managing metallic foreign bodies which at times may need out-of-the-box thinking, like use of motorized cutting tools.

\section{Abbreviations}

Cm: centimetre; SPC: suprapubic catheter; PUC: per urethral catheter.

\section{Acknowledgements}

Not applicable.

\section{Authors' contributions}

MA, VAG, and PS have made substantial contributions to the conception and design of this manuscript. All three were involved in the data acquisition and jointly drafted the work and revised it as per the need. All three were involved in the manuscript drafting, editing, and review. Manuscript was finally approved by all three contributors. MA, VAG, and PS have agreed to be accountable for the manuscript submitted. All authors read and approved the final manuscript.

\section{Funding}

This research received no specific grant from any funding agency in the public, commercial, or not-for-profit sectors.

\section{Availability of data and materials}

Data sharing is not applicable to this article as no datasets were generated or analysed during the current study.

Ethics approval and consent to participate

Not applicable. 


\section{Consent for publication}

Written informed consent was obtained from the patients for publication of this case reports and accompanying images.

\section{Competing interests}

The authors declare that they have no competing interests.

Received: 23 April 2020 Accepted: 20 July 2020

Published online: 09 October 2020

\section{References}

1. Choudhary SK, Taraphdar T, Thomas AJ (2014) Penile strangulation with metallic objects in adults: 2 case reports and literature review. Emerg Med (Los Angel) 4:214

2. Bhat AL, Kumar A, Mathur SC, Gangwal KC (1991) Penile strangulation. Br J Urol 68:618-621

3. Yoshida T, Watanabe D, Minowa T, Yamashita A, Miura K, Mizushima A (2019) Penile strangulation intentionally using a rubber band to prevent the development of penile cancer. Urol Case Rep 27:101003

4. Trivedi S, Attam A, Kerketa A, Daruka N, Behre B, Agarwal A et al (2013) Penile incarceration with metallic foreign bodies: management and review of literature. Curr Urol 7:45-50

5. Silberstein J, Grabowski J, Lakin C, Goldstein I (2008) Penile constriction devices: case report, review of the literature, and recommendations for extrication. J Sex Med 5:1747-1757
6. Gautier M (1775) Observation d'un entanglement et des testicules et de la verge, occasione par le passage d'un briquette. J Med Chir Pharmacol 3:358

7. Sarkar D, Gupta S, Maiti K, Jain P, Pal DK (2019) Penile strangulation by different objects and its removal by the modified string method: management of four cases with review of literature. Urol Ann 11:1-5

8. Ichaoui H, Sallami S, Samet A, Bokal Z, Touinsi H (2018) Strangulation of the penis by a metallic ring: prevention is better than cure. Case Rep Urol 2018:1725752

9. Stuppler SA, Walker JG, Kandzari SJ, Milam DF (1973) Incarceration of penis by foreign body. Urology 2:308-309

10. Tiwari VS, Razdan JL, Yadav VN (1977) Strangulation of the penis by a metallic nut. Int Surg 62:558-560

11. Vahasarja VJ, Hellstrom PA, Serlo W, Kontturi MJ (1993) Treatment of penile incarceration by the string method: 2 case reports. J Urol 149:372-373

12. Noh J, Kang TW, Heo T, Kwon DD, Park K, Ryu SB (2004) Penile strangulation treated with the modified string method. Urology 64:591

13. Singh I, Suman D, Gupta S, Garg G (2018) Penile strangulation by multiple steel ball bearings: desperate situation-desperate measures. BMJ Case Rep 2018:bcr2018227586

\section{Publisher's Note}

Springer Nature remains neutral with regard to jurisdictional claims in published maps and institutional affiliations.

\section{Submit your manuscript to a SpringerOpen ${ }^{\circ}$ journal and benefit from:}

- Convenient online submission

Rigorous peer review

- Open access: articles freely available online

- High visibility within the field

- Retaining the copyright to your article

Submit your next manuscript at springeropen.com 